\title{
Majorana dark matter in a classically scale invariant model
}

\author{
Sanjin Benić ${ }^{a}$ and Branimir Radovčić ${ }^{a, b}$ \\ ${ }^{a}$ Department of Physics, University of Zagreb, \\ Bijenička c. 32, 10002 Zagreb, Croatia \\ ${ }^{b}$ Max-Planck-Institut für Kernphysik, \\ Saupfercheckweg 1, 69117 Heidelberg, Germany \\ E-mail: sanjinb@phy.hr, radovcic@mpi-hd.mpg.de
}

ABStRACT: We analyze a classically scale invariant extension of the Standard Model with a dark gauge $\mathrm{U}(1)_{X}$ broken by a doubly charge scalar $\Phi$ leaving a remnant $Z_{2}$ symmetry. Dark fermions are introduced as dark matter candidates and for anomaly reasons we introduce two chiral fermions. Due to classical scale invariance, bare mass term that would mix these two states is absent and they end up as stable Majorana fermions $N_{1}$ and $N_{2}$. We calculate cross sections for $N_{a} N_{a} \rightarrow \phi \phi, N_{a} N_{a} \rightarrow X^{\mu} \phi$ and $N_{2} N_{2} \rightarrow N_{1} N_{1}$ annihilation channels. We put constraints to the model from the Higgs searches at the LHC, dark matter relic abundance and dark matter direct detection limits by LUX. The dark gauge boson plays a crucial role in the Coleman-Weinberg mechanism and has to be heavier than $680 \mathrm{GeV}$. The viable mass region for dark matter is from $470 \mathrm{GeV}$ up to a few $\mathrm{TeV}$. In the case when the two Majorana fermions have different masses, two dark matter signals at direct detection experiments could provide a distinctive signature of this model.

Keywords: Beyond Standard Model, Higgs Physics

ArXiv EPRINT: 1409.5776 


\section{Contents}

1 Introduction 1

2 The model 2

3 Annihilation cross sections $\quad 4$

4 Dark matter constraints $\quad 6$

4.1 Freeze-out and relic abundance 6

$\begin{array}{lll}4.2 & \text { Direct and indirect detection } & 7\end{array}$

5 Results $\quad 8$

5.1 The case of degenerate masses 8

$\begin{array}{lr}5.2 \text { The case of non-degenerate masses } & 10\end{array}$

6 Conclusions 11

\section{Introduction}

With the recent discovery of the Higgs boson at the LHC $[1,2]$ comes the hierarchy problem. The null results in the first LHC run for supersymmetry, large extra dimensions and other popular theoretical resolutions of the hierarchy problem invites us to think about alternatives. One of the possible alternatives is the classical scale invariance, first discussed by Bardeen [3]. In this scenario, all masses are generated through the quantum breaking of scale invariance. Such a mechanism is already present in Nature: in the chiral limit the QCD part of the Standard Model (SM) Lagrangian is scale invariant and the proton mass is determined by quantum effects. Thus, the main idea is to promote classical scale invariance to a general principle of Nature and apply it also to the electroweak (EW) theory.

In perturbation theory, radiative mass generation can be accomplished through the Coleman-Weinberg (CW) mechanism [4] with the Higgs boson playing the role of the scalon - the pseudo Goldstone boson of the broken scale invariance [5]. Since the CW mechanism is not possible in the SM due to the large top quark contribution, a number of classical scale invariant theories that go beyond the SM [6-9], in several directions [10-29] were presented.

Nowadays, there is an ample astronomical and cosmological evidence that around $27 \%$ of the Universe is made of dark matter (DM). Its relic abundance points to the annihilation cross section of cold DM around the EW scale, making the Weakly Interacting Massive Particles (WIMPs) the leading DM candidates. This is another important hint for the physics beyond the SM which stimulates tremendous present and future experimental efforts in the direct, indirect and accelerator searches for DM. 
In a scale invariant setup any dimensionfull quantity arises from a common scale. It is then tempting to establish a connection between the EW scale and a priori unrelated DM scale by generating both through the $\mathrm{CW}$ mechanism. This exciting possibility was investigated in a number of papers [9, 30-42], and is the main motivation for our work. The model used here is described by a dark $\mathrm{U}(1)_{X}$ gauge group with a doubly charged scalar $\Phi$ coupled to the SM via the Higgs portal. In this construction the scale is generated through quantum corrections in the dark sector and transmitted to the EW sector through the Higgs portal. The dark sector contains two Majorana fermions $N_{1}$ and $N_{2}$. Due to the remnant $Z_{2}$ symmetry both of these particles are stable and are therefore DM candidates.

We extend the investigation of this model initiated in [38] in several directions. We take into account that both Majorana fermions contribute to the DM relic abundance. Besides the annihilation of $N_{1,2}$ into the $\phi \phi$ channel we also include the $X^{\mu} \phi$ channel. We use the recent LUX constraints for spin independent direct detection [43]. Finally, we discuss two cases for the ratio of the Majorana fermions masses. We find that the DM particle lies within the range of $470 \mathrm{GeV}$ up to a few $\mathrm{TeV}$. The lower limit is set by the LHC constraints on the mixing angle between the dark scalar and the SM scalar. The upper limit comes from the estimated limitations set by perturbation theory. We find that the allowed parameter region of the model is also constrained by the direct detection limits set by LUX. The DM relic abundance is saturated by moderate values of the dark Yukawas and dark gauge coupling. The values for the spin independent cross section that are presently allowed are in the reach of future experiments. The sensitivity of the forthcoming XENON1T [44] and LZ [45] experiments offers excellent perspective for testing this model. In the case when the two Majorana fermions have different masses, two signals at direct detection experiments provide a distinctive signature for this model.

\section{The model}

The relevant part of the model Lagrangian is given as

$$
\begin{aligned}
\mathcal{L}= & i \bar{N}_{L}\left(\not \partial-i g_{X} X\right) N_{L}-\frac{y_{1}}{2}\left(\bar{N}_{L} N_{L}^{c} \Phi+\text { h. c. }\right) \\
& +i \bar{N}_{R}\left(\not \partial-i g_{X} \not X\right) N_{R}-\frac{y_{2}}{2}\left(\bar{N}_{R} N_{R}^{c} \Phi+\text { h. c. }\right) \\
& +\left|\left(\partial_{\mu}-2 i g_{X} X_{\mu}\right) \Phi\right|^{2}-\frac{1}{4} X_{\mu \nu} X^{\mu \nu}-\frac{1}{2} \sin \epsilon B_{\mu \nu} X^{\mu \nu}-V(H, \Phi) .
\end{aligned}
$$

where $N_{L, R}$ are left and right chiral fields with the same charge $g_{X}$ as required by anomaly cancellation, and Yukawa couplings $y_{1,2}$ to the doubly $\mathrm{U}(1)_{X}$ charged scalar $\Phi$. The dark gauge $X$-boson couples to the $\mathrm{U}(1)_{Y}$ SM gauge field via the kinetic mixing parameter $\sin \epsilon$ [46]. This will lead to the coupling of the $X$-boson to a pair of SM particles with the interactions suppressed by the mixing parameter $\sin \epsilon$ [49]. After spontaneous symmetry breaking in the dark sector we end up with two Majorana fields

$$
N_{1}=N_{L}+N_{L}^{c}, \quad N_{2}=N_{R}+N_{R}^{c}
$$


The scalar potential is given as

$$
V(H, \Phi)=\frac{\lambda_{H}}{2}\left(H^{\dagger} H\right)^{2}+\frac{\lambda_{\Phi}}{2}\left(\Phi^{\dagger} \Phi\right)^{2}+\lambda_{P}\left(H^{\dagger} H\right)\left(\Phi^{\dagger} \Phi\right),
$$

where $H$ is the SM Higgs field, and $\Phi$ is a singlet under the SM group.

The Lagrangian in (2.1)-(2.3) is classically scale invariant. Therefore all the masses in the model will be generated dynamically. This will be accomplished by a CW mechanism, which is a perturbative version of the QCD-like mass generation. The breaking of scale symmetry is obtained by balancing the one-loop effective potential to its tree level counterpart.

We assume vacuum expectation values (vevs) for both scalars

$$
H=\left(\begin{array}{c}
H^{+} \\
\frac{1}{\sqrt{2}}\left(v_{H}+h^{\prime}+i G\right)
\end{array}\right), \quad \Phi=\frac{1}{\sqrt{2}}\left(v_{\Phi}+\phi^{\prime}+i J\right) .
$$

In this work we use the GW framework [5] which imposes that the potential at the classical level is flat along a particular direction in field space. We first define a vector from scalar fields with non-vanishing vevs given in the polar coordinates as

$$
\left(\begin{array}{l}
v_{H}+h^{\prime} \\
v_{\Phi}+\phi^{\prime}
\end{array}\right)=r\left(\begin{array}{c}
\sin \theta \\
\cos \theta
\end{array}\right)
$$

The rewritten tree-level potential reads

$$
V(r)=r^{4}\left(\frac{\lambda_{H}}{8} \cos ^{4} \theta+\frac{\lambda_{\Phi}}{8} \sin ^{4} \theta+\frac{\lambda_{P}}{4} \sin ^{2} \theta \cos ^{2} \theta\right) .
$$

The vanishing bracket defines the flat direction

$$
\lambda_{H}(\Lambda) \lambda_{\Phi}(\Lambda)-\lambda_{P}^{2}(\Lambda)=0,
$$

given at some common scale $\Lambda$ by the angle $\theta$

$$
\sin ^{2} \theta=-\frac{\lambda_{P}}{\lambda_{H}-\lambda_{P}} .
$$

Minimizing the potential we can also obtain the following relation between the vevs

$$
\frac{v_{H}^{2}}{v_{\Phi}^{2}}=-\frac{\lambda_{P}}{\lambda_{H}} .
$$

Scalar mass eigenstates are given by

$$
\left(\begin{array}{l}
h \\
\phi
\end{array}\right)=\left(\begin{array}{cc}
\cos \theta & -\sin \theta \\
\sin \theta & \cos \theta
\end{array}\right)\left(\begin{array}{l}
h^{\prime} \\
\phi^{\prime}
\end{array}\right)
$$

where the mixing angle $\theta$ is given in (2.8). At the tree level the particle masses are

$$
\begin{aligned}
m_{h}^{2} & =\left(\lambda_{H}-\lambda_{P}\right) v_{H}^{2}, \\
m_{X}^{2} & =4 g_{X}^{2} v_{\Phi}^{2}, \\
m_{N_{a}} & =\frac{y_{a}}{\sqrt{2}} v_{\Phi},
\end{aligned}
$$

while $\phi$ remains massless at the tree level. 
The common scale in the theory is generated by radiative corrections. Then, the splitting in the spectra is solely due to the different values of the dimensionless couplings in the model. The quantum loop corrections are built on top of the flat direction [5]. The one-loop scalar potential reads $[5,9]$

$$
\delta V(r)=A r^{4}+B r^{4} \log \left(\frac{r^{2}}{\Lambda^{2}}\right)
$$

where $[9,38]$

$$
\begin{aligned}
A= & \frac{1}{64 \pi^{2} v_{r}^{4}}\left\{m_{h}^{4}\left(-\frac{3}{2}+\log \frac{m_{h}^{2}}{v_{r}^{2}}\right)+6 m_{W}^{4}\left(-\frac{5}{6}+\log \frac{m_{W}^{2}}{v_{r}^{2}}\right)\right. \\
& +3 m_{Z}^{4}\left(-\frac{5}{6}+\log \frac{m_{Z}^{2}}{v_{r}^{2}}\right)+3 m_{X}^{4}\left(-\frac{5}{6}+\log \frac{m_{X}^{2}}{v_{r}^{2}}\right) \\
& -12 m_{t}^{4}\left(-1+\log \frac{m_{t}^{2}}{v_{r}^{2}}\right)-2 m_{N_{1}}^{4}\left(-1+\log \frac{m_{N_{1}}^{2}}{v_{r}^{2}}\right) \\
& \left.-2 m_{N_{2}}^{4}\left(-1+\log \frac{m_{N_{2}}^{2}}{v_{r}^{2}}\right)\right\}, \\
B= & \frac{1}{64 \pi^{2} v_{r}^{4}}\left(m_{H}^{4}+6 m_{W}^{4}+3 m_{Z}^{4}+3 m_{X}^{4}-12 m_{t}^{4}-2 m_{N_{1}}^{4}-2 m_{N_{2}}^{4}\right),
\end{aligned}
$$

and $v_{r}$ is the vev of the field $r$. The mass for the scalar $\phi$, the so-called scalon, is generated purely by radiative corrections. In the GW framework [5] this is

$$
m_{\phi}^{2}=\frac{1}{8 \pi^{2} v_{H}^{2} \sin ^{2} \theta}\left(m_{H}^{4}+6 m_{W}^{4}+3 m_{Z}^{4}+3 m_{X}^{4}-12 m_{t}^{4}-2 m_{N_{1}}^{4}-2 m_{N_{2}}^{4}\right) .
$$

Due to the quantum numbers of $N_{L, R}$ a Dirac mass term mixing the two components would be possible. However, this would be in contradiction with the principle of classical scale invariance. Thus, both Majorana fermions are stable due to the respective remnant $Z_{2}$ symmetry making them the DM candidates in this model. Related models of Majorana DM have been discussed recently. In [47] the heavier Majorana fermion is unstable, while in [48] the mixing between $N_{L, R}$ was forbidden by a $Z_{2}$. Due to the couplings to the SM particles via the kinetic mixing term in (2.1) the dark X-boson is unstable. For it to decay sufficiently quickly at the DM decoupling, the kinetic mixing parameter takes on the value larger than roughly $\sin \epsilon \gtrsim 10^{-8}$. For possible $X$-boson LHC phenomenology, including a study of the constraints on $\sin \epsilon$, see [49]. LHC phenomenology of the scalar $\phi$ is similar to the scalar singlet extensions of the SM [36, 40, 42].

\section{Annihilation cross sections}

In order to calculate the dark matter relic abundance we need to know the $N_{1,2}$ annihilation cross sections. In this work we will assume $m_{N_{2}} \geq m_{N_{1}}$ and consider the following channels

$$
\begin{aligned}
& N_{a} N_{a} \rightarrow \phi \phi, h \phi, h h, \\
& N_{a} N_{a} \rightarrow X \phi, X h, \\
& N_{2} N_{2} \rightarrow N_{1} N_{1},
\end{aligned}
$$

as shown on figure 1. 

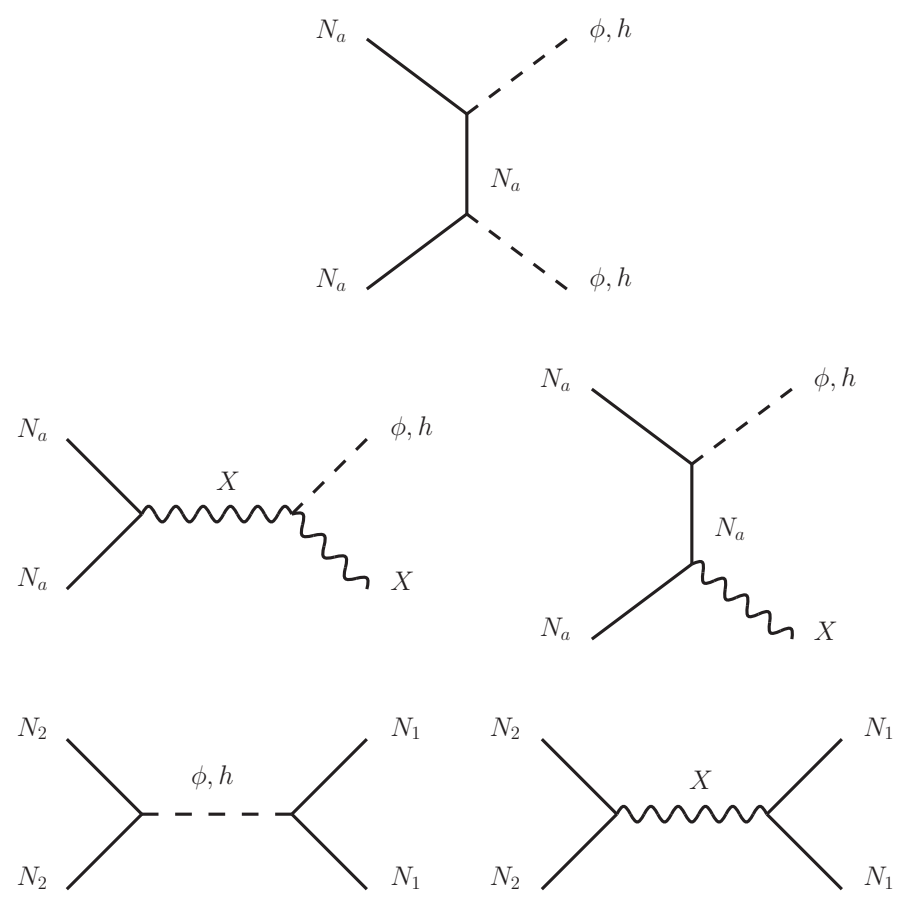

Figure 1. Annihilation processes of Majorana dark matter $N_{1}$ and $N_{2}$ considered in this work.

The DM annihilation to SM particles is suppressed by a small portal coupling, the only communication between the dark and the SM sector. We have checked that those channels don't play a numerically significant role. We expand the cross sections in powers of the relative velocity $v$, and show only the dominant components, that is, the annihilation into the dark sector.

The cross section for the $N_{a} N_{a} \rightarrow \phi \phi$ channel is given as

$$
\sigma\left(N_{a} N_{a} \rightarrow \phi \phi\right) v=\sigma_{a a \phi \phi}^{p} v^{2}+\mathcal{O}\left(v^{4}\right)
$$

where

$$
\sigma_{a a \phi \phi}^{p}=\frac{y_{i}^{4} \cos ^{4} \theta}{96 \pi} \frac{m_{N_{a}}^{2}\left(9 m_{N_{a}}^{4}-8 m_{N_{a}}^{2} m_{\phi}^{2}+2 m_{\phi}^{4}\right)}{\left(m_{\phi}^{2}-2 m_{N_{a}}^{2}\right)^{4}} \sqrt{1-\frac{m_{\phi}^{2}}{m_{N_{a}}^{2}}} .
$$

The cross section for the $N_{a} N_{a} \rightarrow X \phi$ channel is given as

$$
\sigma\left(N_{a} N_{a} \rightarrow X \phi\right) v=\sigma_{a a \phi X}^{s}+\sigma_{a a \phi X}^{p} v^{2}+\mathcal{O}\left(v^{4}\right),
$$

where

$$
\begin{aligned}
\sigma_{a a \phi X}^{s}= & \frac{g_{X}^{6} \cos ^{2} \theta}{16 \pi} v_{\Phi}^{2} \frac{m_{X}^{4}+\left(m_{\phi}^{2}-4 m_{N_{a}}^{2}\right)^{2}-2 m_{X}^{2}\left(m_{\phi}^{2}+4 m_{N_{a}}^{2}\right)}{m_{N_{a}}^{2} m_{X}^{6}} \\
& \times \sqrt{\frac{m_{X}^{4}+\left(m_{\phi}^{2}-4 m_{N_{a}}^{2}\right)^{2}-2 m_{X}^{2}\left(m_{\phi}^{2}+4 m_{N_{a}}^{2}\right)}{m_{N_{a}}^{4}}}
\end{aligned}
$$


and

$$
\begin{aligned}
\sigma_{a a \phi X}^{p}= & \frac{g_{X}^{2}}{768 \pi} \frac{\cos ^{2} \theta}{m_{N_{a}}^{2} m_{X}^{6}\left(m_{X}^{2}-4 m_{N_{a}}^{2}\right)^{2}\left(-4 m_{N_{a}}^{2}+m_{\phi}^{2}+m_{X}^{2}\right)^{4}}\left\{-8 g_{X}^{4} v_{\Phi}^{2}\left(-4 m_{N_{a}}^{2}+m_{\phi}^{2}+m_{X}^{2}\right)^{4}\right. \\
& \times\left[384 m_{N_{a}}^{8}-96 m_{N_{a}}^{6}\left(5 m_{\phi}^{2}+7 m_{X}^{2}\right)+8 m_{N_{a}}^{4}\left(12 m_{\phi}^{2}+6 m_{\phi}^{2} m_{X}^{2}+43 m_{X}^{4}\right)\right. \\
& \left.-2 m_{N_{a}}^{2}\left(24 m_{\phi}^{4} m_{X}^{2}-37 m_{\phi}^{2} m_{X}^{4}+59 m_{X}^{6}\right)+5 m_{X}^{4}\left(m_{\phi}^{2}-m_{X}^{2}\right)^{2}\right] \\
& -16 \sqrt{2} y_{a} g_{X}^{2} v_{\Phi} m_{N_{a}} m_{X}^{2}\left(4 m_{N_{a}}^{2}-m_{X}^{2}\right)\left(-4 m_{N_{a}}^{2}+m_{\phi}^{2}+m_{X}^{2}\right)^{2} \\
& \times\left[128 m_{N_{a}}^{8}-96 m_{N_{a}}^{6}\left(m_{\phi}^{2}+m_{X}^{2}\right)+8 m_{N_{a}}^{4}\left(3 m_{\phi}^{4}+2 m_{\phi}^{2} m_{X}^{2}+13 m_{X}^{4}\right)\right. \\
& \left.-2 m_{N^{2}}^{2}\left(m_{\phi}^{2}+m_{X}^{2}\right)\left(m_{\phi}^{4}-2 m_{\phi}^{2} m_{X}^{2}+9 m_{X}^{4}\right)-m_{X}^{4}\left(m_{\phi}^{2}-m_{X}^{2}\right)^{2}\right] \\
& +y_{a}^{2} m_{X}^{4}\left(m_{X}^{2}-4 m_{N_{a}}^{2}\right)^{2}\left[m_{X}^{10}+4 m_{X}^{8}\left(m_{N_{a}}^{2}-m_{\phi}^{2}\right)+2 m_{X}^{6}\left(16 m_{N}^{4}+3 m_{\phi}^{4}\right)\right. \\
& \left.-4 m_{X}^{4}\left(m_{\phi}^{2}-4 m_{N_{a}}^{2}\right)^{2}\left(10 m_{N_{a}}^{2}+m_{\phi}^{2}\right)+m_{X}^{2}\left(m_{\phi}^{2}-4 m_{N_{a}}^{2}\right)^{2}\left(80 m_{N_{a}}^{4}+8 m_{N_{a}}^{2} m_{\phi}^{2}+m_{\phi}^{4}\right)\right] \\
& \left.+4 m_{N_{a}}^{2}\left(m_{\phi}^{2}-4 m_{N_{a}}^{2}\right)^{4}\right\} \sqrt{\frac{m_{X}^{4}+\left(m_{\phi}^{2}-4 m_{N_{a}}^{2}\right)^{2}-2 m_{X}^{2}\left(m_{\phi}^{2}+4 m_{N_{a}}^{2}\right)}{m_{N_{a}}^{4}}} .
\end{aligned}
$$

For simplicity and clarity we do not list the cross sections for annihilations to Higgs bosons, but we include them in our numerical calculations. The cross section for the $N_{2} N_{2} \rightarrow N_{1} N_{1}$ channel is given as

$$
\sigma\left(N_{2} N_{2} \rightarrow N_{1} N_{1}\right) v=\sigma_{2211}^{s}+\sigma_{2211}^{p} v^{2}+\mathcal{O}\left(v^{4}\right)
$$

where

$$
\sigma_{2211}^{s}=\frac{g_{X}^{4}}{4 \pi} \frac{m_{N_{1}}^{2}}{m_{X}^{4}} \sqrt{1-\frac{m_{N_{1}}^{2}}{m_{N_{2}}^{2}}}
$$

and

$$
\begin{aligned}
\sigma_{2211}^{p}= & \frac{1}{192 \pi}\left\{\frac { 2 g _ { X } ^ { 4 } } { ( m _ { X } ^ { 2 } - 4 m _ { N _ { 2 } } ^ { 2 } ) ^ { 2 } ( m _ { N _ { 2 } } ^ { 2 } - m _ { N _ { 1 } } ^ { 2 } ) } \left[m_{N_{1}}^{4}\left(240 m_{N_{2}}^{4}-120 m_{N_{2}}^{2} m_{X}^{2}+23 m_{X}^{4}\right)\right.\right. \\
& \left.-4 m_{N_{1}}^{2}\left(48 m_{N_{2}}^{6}-24 m_{N_{2}}^{4} m_{X}^{2}+7 m_{N_{2}}^{2} m_{X}^{4}\right)+8 m_{N_{2}}^{4} m_{X}^{4}\right] \\
& \left.+\frac{3 y_{1}^{2} y_{2}^{2} \cos ^{4} \theta}{\left(m_{\phi}^{2}-4 m_{N_{2}}^{2}\right)^{2}}\left(m_{N_{2}}^{2}-m_{N_{1}}^{2}\right)\right\} \sqrt{1-\frac{m_{N_{1}}^{2}}{m_{N_{2}}^{2}}} .
\end{aligned}
$$

\section{Dark matter constraints}

\subsection{Freeze-out and relic abundance}

In this model we have two DM candidates, $N_{1}$ and $N_{2}$. If $N_{1}$ is much lighter than $N_{2}$, it could not annihilate to dark scalars and dark gauge bosons. It could only annihilate directly to the SM particles, with too small cross sections to satisfy the DM relic abundance constraint. Only possible solution would be for $N_{1}$ to decouple while it is relativistic which would put strong bounds on its mass, around $m_{N_{1}}<1 \mathrm{eV}$. That would require a tiny 
Yukawa coupling $y_{1}$ and it would not be in line with the idea that all new particle masses are at the scale generated by quantum effects. That is why we take the masses of $N_{1}$ and $N_{2}$ of the same order of magnitude.

We will focus on two mass hierarchies, the degenerate mass case where $m_{N_{2}}=m_{N_{1}}$ and non-degenerate mass case with $m_{N_{2}}=1.5 m_{N_{1}}$. In the degenerate case the annihilation of $N_{1}$ and $N_{2}$ into each other is not important for the DM relic abundance. In principle, with different masses, the channel $N_{2} N_{2} \rightarrow N_{1} N_{1}$ mixes the two coupled Boltzmann equations, but if the mass splitting is large enough, the DM freeze-out is sequential so that the decoupling of $N_{1}$ happens after the decoupling of $N_{2}$. Both particles leave the thermal bath at respective temperatures $T_{f}^{a}$ given by $m_{N_{a}} / T_{f}^{a}=x_{f}^{a} \simeq 25$. Lets set $\Delta x$ as the width of the freeze-out region. Then the condition on $N_{2}-N_{1}$ mass ratio to have a sequential freeze-out is

$$
\frac{m_{N_{2}}}{m_{N_{1}}} \gtrsim 1+\frac{\Delta x}{x_{f}^{a}} .
$$

With typical values of $\Delta x \simeq 5$ and $x_{f}^{a} \simeq 25$ we have $m_{N_{2}} \gtrsim 1.2 m_{N_{1}}$.

The number density over entropy density today, $Y_{\infty}^{a}=n_{N_{a}} / s_{0}$ is approximately given by the formula $[50,51]$

$$
Y_{\infty}^{a}=\frac{3.79 x_{f}^{a}}{\sqrt{g_{*}} m_{P l} m_{N_{a}}\left(\sigma_{a a X \phi}^{s}+\delta_{a 2} \sigma_{2211}^{s}+\frac{3}{x_{f}^{a}}\left(\sigma_{a a \phi \phi}^{p}+\sigma_{a a X \phi}^{p}+\delta_{a 2} \sigma_{2211}^{p}\right)\right)},
$$

where $g_{*}=86.25$ is the number of relativistic degrees of freedom at $T_{f}$ and $m_{P l}=1.22 \times$ $10^{19} \mathrm{GeV}$ is the Planck mass. The observed dark matter relic abundance of the Universe is

$$
\Omega_{\mathrm{DM}} h^{2}=\frac{\rho_{N_{1}}+\rho_{N_{2}}}{\rho_{c r}}=\frac{m_{N_{1}} n_{N_{1}}+m_{N_{2}} n_{N_{2}}}{\rho_{c r}} .
$$

\subsection{Direct and indirect detection}

It is possible that our DM candidates $N_{1}$ and $N_{2}$ are detected by elastic scattering of a nucleus at the DM direct detection experiments. In our model the interaction with the nucleon is governed by the $t$-channel exchange of scalars $h$ and $\phi$ (see figure 2) The spin independent cross section for direct detection is given by [53]

$$
\sigma_{\mathrm{SI}}=\frac{y_{a}^{2}}{2 \pi} \frac{m_{p}^{4}}{v_{H}^{2}} f^{2} \sin ^{2} \theta \cos ^{2} \theta\left(\frac{1}{m_{\phi}^{2}}-\frac{1}{m_{h}^{2}}\right)^{2},
$$

where $m_{p}$ is the proton mass and $f=0.35$ [54] parameterizes the nuclear matrix element.

We use the LUX published limits [43] on the direct detection cross section. We take into account the fact that we have two DM particles contributing to the relic abundance with different number densities and therefore different event rates. The modified LUX constraint scales as the ratio of the number density of the particular DM component, $n_{a}$, and the number density for which this component would saturate the DM relic abundance.

DM annihilations in the high density regions of the Universe could lead to indirect detection signals $[55,56]$. In our case, $N_{1}$ and $N_{2}$ cannot annihilate directly into photons, so 


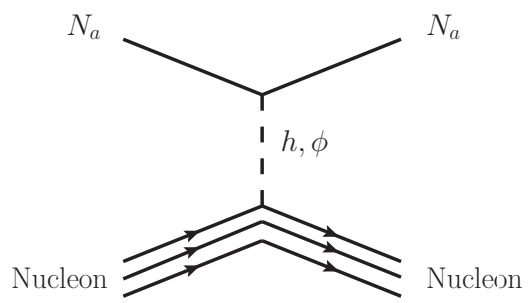

Figure 2. Scattering of Majorana dark matter $N_{a}$ of a nucleon.

they cannot produce gamma lines. If the positron excess measured by PAMELA and AMS02 comes from DM annihilations, the cross section for DM annihilations has to be larger than in the usual WIMP scenario [56]. Thus, our model cannot explain the positron excess measured by PAMELA or AMS-02. In principle, DM annihilations will produce fluxes of SM particles, including the flux of continuum gamma rays and antiprotons. However, searches for all such annihilations products are not yet sensitive enough to reach the typical values of the WIMP cross section for DM masses above $1 \mathrm{TeV}[56]$ as found in our model (see the following section). Similar conclusions were reached in [14]. We conclude that at the moment, our model is not constrained by present DM indirect searches.

\section{$5 \quad$ Results}

In the following we show the predictions of this model for several quantities of interest. There are six new parameters in the model: the gauge coupling $g_{X}$, the two Yukawa couplings $y_{1}$ and $y_{2}$, and the three quartic scalar couplings $\lambda_{P}, \lambda_{H}$ and $\lambda_{\Phi}$. These are to be constrained by the Higgs boson mass $m_{h}=125 \mathrm{GeV}$ and $v e v v_{H}=246 \mathrm{GeV}$ and the DM relic abundance $\Omega_{\mathrm{DM}} h^{2}=0.1187(17)$ [52] leaving three undetermined parameters.

We will show our results by fixing the mass ratio of the Majorana fermions (or, equivalently, the ratio of $y_{a}$ 's) and varying the scalon $\phi$ and the dark $X$-boson mass in a region set as follows. A light scalon $\phi$ is excluded in our model by the LEP Higgs searches providing a lower bound of $114 \mathrm{GeV}$. In addition to the large top quark contribution to the radiatively generated scalon mass $m_{\phi}$ in (2.17), there are also contributions from DM Majorana fermions $N_{1}$ and $N_{2}$ that need to be overcome by the dark $X$-boson contributions for the CW mechanism to work. This sets a lower limit on dark $X$-boson mass. The parameter space we will cover starts from the initial values $m_{\phi}=114 \mathrm{GeV}$ and $m_{X}=600 \mathrm{GeV}$.

Our results also take into account the current LHC data providing an upper bound on the scalar mixing angle $\sin \theta<0.37$ [36, 40, 42]. In our calculations we will also include the direct detection constraint discussed in the previous section.

\subsection{The case of degenerate masses}

Here we focus on the scenario

$$
m_{N_{1}}=m_{N_{2}},
$$

where both Majorana fermions contribute equally to the observed DM relic abundance. The main result is the Majorana DM mass shown on the left panel of figure 3 in the 

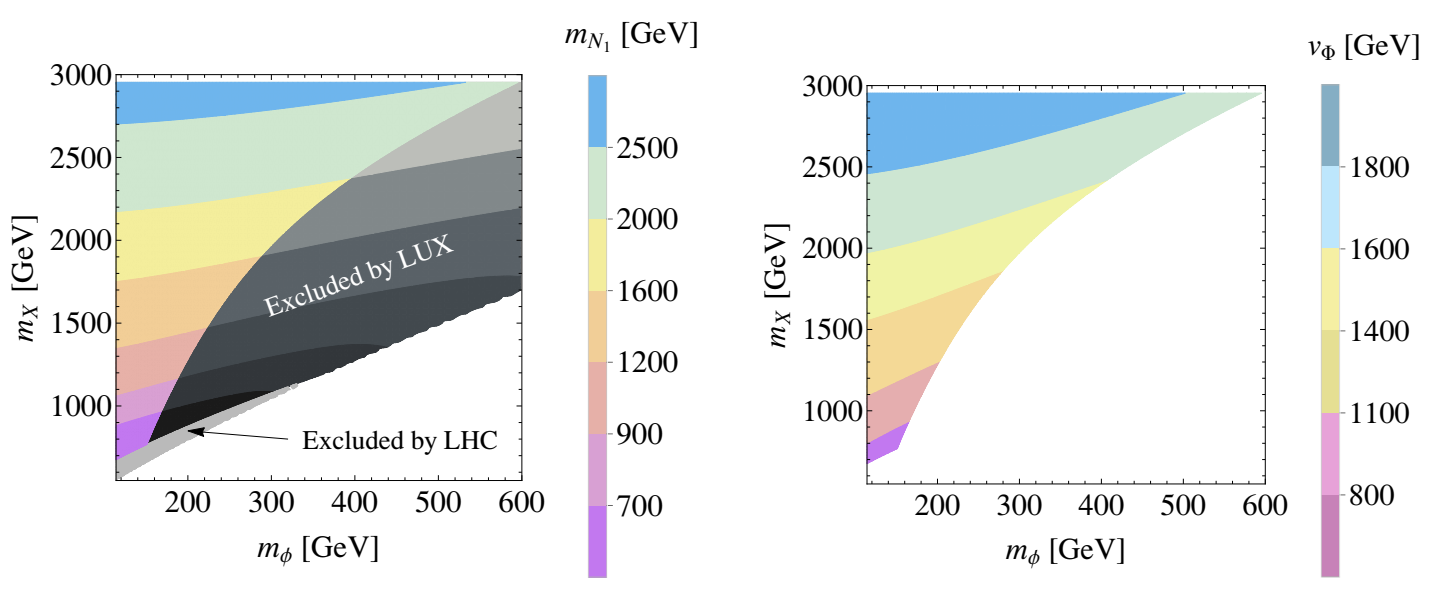

Figure 3. Left (right) panel: mass of the DM Majorana fermion $m_{N_{1}}$ (vev of the $\mathrm{U}(1)_{X}$ charged scalar $v_{\Phi}$ ) in the $m_{\phi}-m_{X}$ plane for the degenerate mass case. We impose the LHC bound on $\sin \theta$ and the LUX experiment constraints.

$m_{\phi}-m_{X}$ plane. The region of low masses $\left(m_{N_{1}} \lesssim 470 \mathrm{GeV}\right)$ is excluded by the current LHC bound on the scalar mixing angle $\sin \theta<0.37$. This also sets the lower bound on the dark $X$-boson to be $680 \mathrm{GeV}$. Due to its cardinal role in the CW mechanism, the dark gauge boson is the heaviest particle in the dark sector. A substantial portion of the parameter space has been excluded by the LUX experiment [43] as depicted by the gray scaled region on the left panel of figure 3. The mass of the Majorana DM candidates $N_{1}$ and $N_{2}$, is from $470 \mathrm{GeV}$ to a few $\mathrm{TeV}$.

In general, we find moderate values of the dark gauge and the Yukawa couplings. The Yukawa coupling $y_{1}$ can be deduced by combining the results from figure 3 with (2.13) to lie in the region $1.0 \lesssim y_{1} \lesssim 2.0$ covered by the corresponding $m_{\phi}$ and $m_{X}$ values. The gauge coupling is shown on the left panel of figure 4 . The behavior of $g_{X}$ can be understood as follows: a small scalar mixing angle leads to a small portal coupling $\lambda_{P}$. In order to keep the Higgs mass fixed, this leads to a large vev $v_{\Phi}$ of the scalon in (2.9), typically by an order of magnitude larger than the EW scale. This vev, shown on right panel of figure 3, sets the scale of the DM annihilation processes to the hidden sector. Therefore, since we know that the DM cross section should be of the order of the EW scale, we need appreciable dark gauge $g_{X}$ and Yukawa $y_{a}$ couplings to compensate the large vev. In addition, the $\mathrm{CW}$ mechanism favors large gauge couplings, but, at the same time, disfavors large Yukawa couplings, creating some tension between the two. This is the reason behind the non-monotonous behavior of $g_{X}$ on the left panel of figure 4.

On figure 5 we show the direct detection cross section $\sigma_{\mathrm{SI}}$ as deduced from (4.4). The direct detection proceeds through the Higgs portal and is therefore proportional to the mixing angle $\sin ^{2} \theta$. However, the moderate values of the Yukawa coupling yield $\sigma_{\mathrm{SI}}$ already within the range of the LUX experiment [43]. The obtained result is largely independent of the $X$-boson mass. With the future XENON1T and LZ experiments there is an excellent opportunity to sweep the entire parameter space of the model except around $m_{\phi}=m_{h}$ where $\sigma_{\mathrm{SI}}$ drops to zero. 

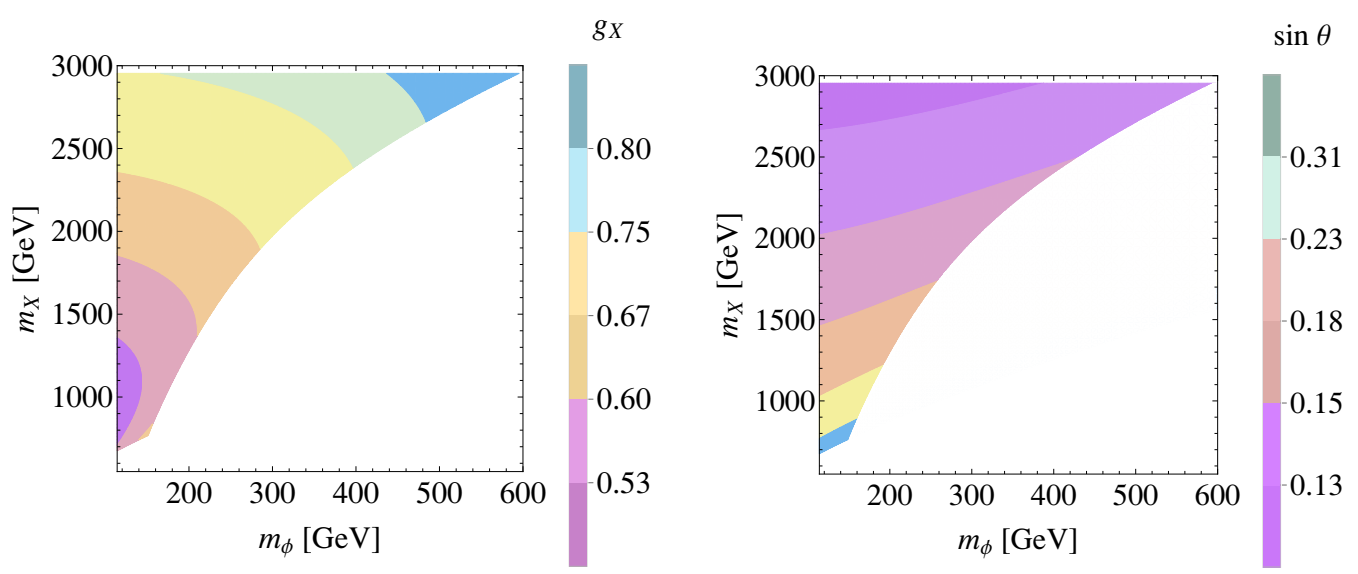

Figure 4. Left (right) panel: the $\mathrm{U}(1)_{X}$ gauge coupling $g_{X}$ (scalar mixing angle $\sin \theta$ ) in the $m_{\phi}-m_{X}$ plane for the degenerate mass case. We impose the LHC bound on $\sin \theta$ and the LUX experiment constraints.

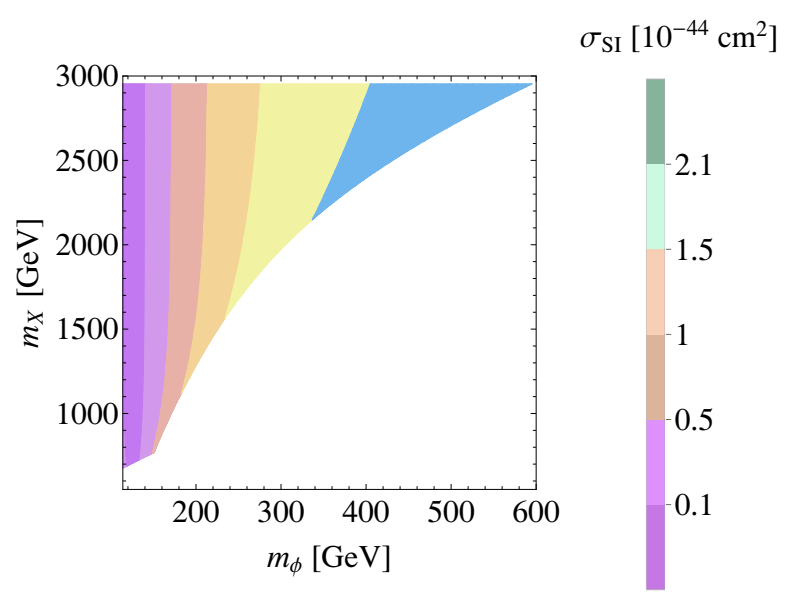

Figure 5. The direct detection cross section $\sigma_{\mathrm{SI}}$ in the $m_{\phi}-m_{X}$ plane for the degenerate mass case. We impose the LHC bound on $\sin \theta$ and the LUX experiment constraints.

\subsection{The case of non-degenerate masses}

Here we consider the case where Majorana fermion masses differ, and for definiteness take

$$
m_{N_{2}}=1.5 m_{N_{1}}
$$

so that the DM Majorana fermions decouple sequentially from the heat bath. In this calculation the appearance of the $N_{2} N_{2} \rightarrow N_{1} N_{1}$ annihilation channel has been taken into account. Our main results are summarized in figures 6 and 7. On the left panel of figure 6 we see that the lighter DM Majorana fermion mass $m_{N_{1}}$ spans a similar region as in the case of the degenerate masses. In fact, the minimum mass of the lighter $N_{1}$ is roughly the same as in the degenerate case, so that a fixed scalon mass requires a larger dark $X$-boson mass. 

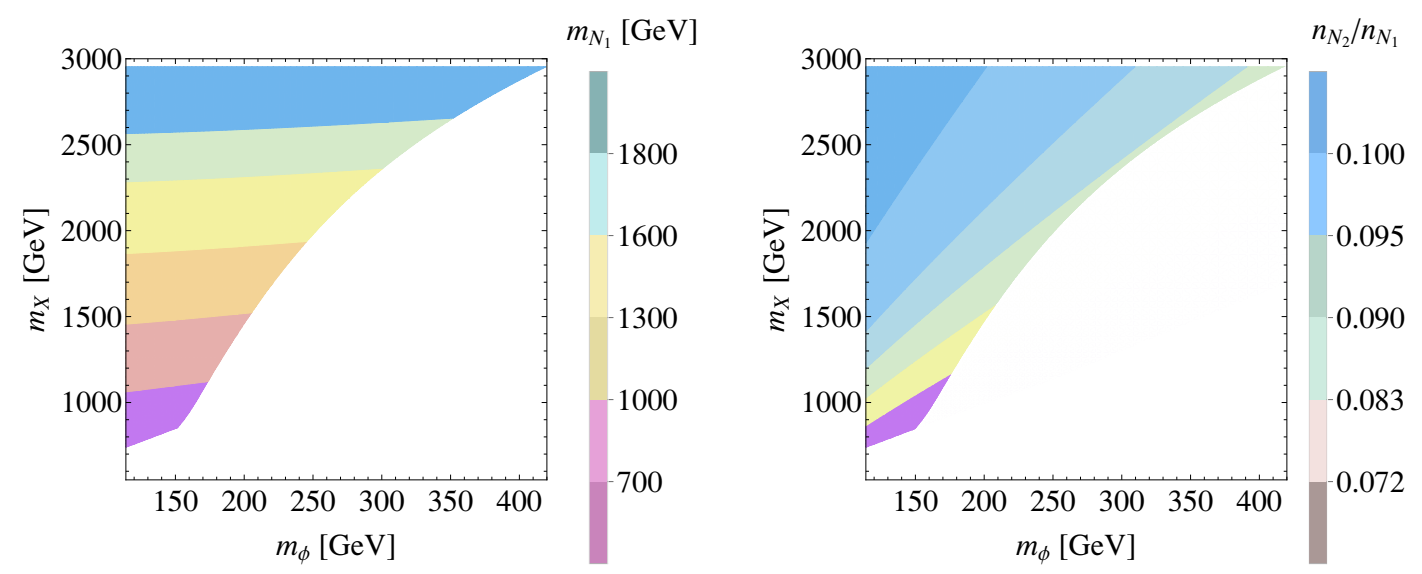

Figure 6. Left (right )panel: mass of the DM Majorana fermion $m_{N_{1}}$ (number density ratio $n_{N_{2}} / n_{N_{1}}$ ) in the $m_{\phi}-m_{X}$ plane for $m_{N_{2}}=1.5 m_{N_{1}}$. We impose the LHC bound on $\sin \theta$ and the LUX experiment constraints.

The main difference with respect to the degenerate mass scenario is that the DM relic abundance is now mainly saturated by $N_{1}$. The $N_{2}$ 's have an extra $N_{2} N_{2} \rightarrow N_{1} N_{1}$ annihilation channel as well as a stronger Yukawa coupling both of which act to decrease the freeze-out abundance $Y_{\infty}^{2}$ according to (4.2). On the right panel of figure 6 we see that the ratio of the number densities of the heavier $N_{2}$ with respect to the lighter $N_{1}$ Majorana fermion is roughly $10 \%$.

On figure 7 we plot the direct detection cross section of $N_{1}$ (left panel) and $N_{2}$ (right panel). We have imposed the LUX constraint on cross sections for $N_{1}$ and $N_{2}$, appropriately modified to take into account the different number densities. As a direct consequence of the number density dominance of the lighter Majorana fermions $N_{1}$ the LUX constraints on $N_{1}$ are more severe in the considered $m_{\phi}-m_{X}$ space. Therefore, this is the constraint imposed on both plots of figure 7 . The attractive feature of this scenario is the two distinct signals in the direct detection experiments. The numerical values on figure 7 indicate that a large portion of the parameter space is testable at future DM searches, such as XENON1T and LZ.

\section{Conclusions}

We have constructed a classically scale invariant model with a dark gauged $\mathrm{U}(1)_{X}$. The $\mathrm{CW}$ mechanism is realized in the dark sector and the scale is transmitted to the SM through the Higgs portal. We have introduced a pair of $\mathrm{U}(1)_{X^{-}}$-charged chiral fermions. Classical scale invariance and gauge invariance leave a separate remnant $Z_{2}$ symmetry for both Majorana fermions after spontaneous symmetry breaking making them the DM candidates in our model. All the masses in the dark sector and the SM sector come from a scale generated dynamically by the CW mechanism. This makes a connection between the DM mass and the EW scale, accounting for the WIMP miracle. 

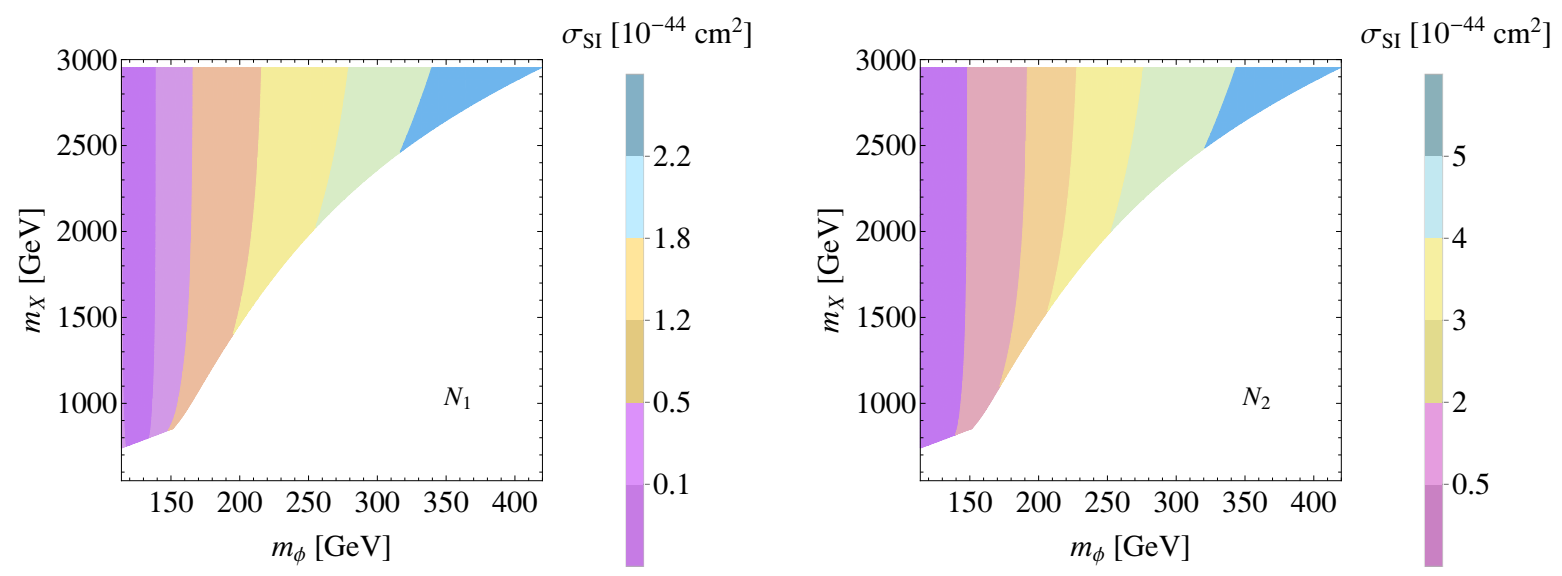

Figure 7. Left (right) panel: Majorana dark matter $N_{1}\left(N_{2}\right)$ direct detection cross section in the $m_{\phi}-m_{X}$ plane for $m_{N_{2}}=1.5 m_{N_{1}}$. We impose the LHC bound on $\sin \theta$ and the LUX experiment constraints.

The model allows for six free parameters, the dark gauge coupling, three couplings in the scalar potential, and two Yukawa couplings in the dark sector. We have constrained the model by the Higgs mass and vev and the observed DM relic abundance. We have also used LHC constraints on the scalar mixing angle and the LUX experiment results on the direct detection of DM. The three undetermined parameters were chosen to be the dark scalar and the dark gauge boson masses, and the ratio of Majorana fermion masses.

We have analyzed two possible cases for fixed ratio of the Majorana fermions masses, the case of equal masses $m_{N_{2}}=m_{N_{1}}$ and the case with the ratio $m_{N_{2}}=1.5 m_{N_{1}}$. In both cases the mass of the Majorana DM can be from $470 \mathrm{GeV}$ to a few $\mathrm{TeV}$. The lower limit is set by the LHC constraints on the mixing angle between the SM and the dark sector scalars, while the upper limit is an estimate from applicability of perturbation theory. The constraints on the model result in moderate values of the dark gauge and the dark Yukawa couplings. In the degenerate mass case both Majorana fermions contribute equally to the DM relic abundance, while in the second case the heavier Majorana fermion $N_{2}$ accounts only for $\sim 10 \%$ of the number density of $N_{1}$.

At the LHC the dark sector can be reached through the Higgs portal. However, due to the large masses in the dark sector the model does not allow for hidden decay channel of the SM Higgs particle. The scalon $\phi$ couples to the SM particles through the scalar mixing with a suppression factor given by the mixing angle $\sin \theta$. LHC data excludes a region of $\sin \theta>0.37$ ruling out the dark $X$-boson masses lower then $680 \mathrm{GeV}$ and Majorana DM masses lower then $470 \mathrm{GeV}$. The next LHC run will further test a part of the parameter space of the model.

The direct detection experiments offer the best prospects to test this model. The interaction with the nucleus proceeds through the Higgs portal, and the key role is played by the dark scalar mass and the Yukawa couplings in the dark sector. Due to the considerable values of the Yukawa couplings, the spin independent cross section with the nucleus is 
of the order of $10^{-44} \mathrm{~cm}^{2}$ which has been reached in the LUX experiment for the DM mass range obtained in this model. While the latest LUX constraints already exclude a substantial region of the parameter space, the planned XENON1T and LZ experiments will be able to sweep a majority of the parameter space. A unique signature for this model would be two distinctive signals in the direct detection experiments due to the presence of two non-degenerate Majorana DM candidates.

\section{Acknowledgments}

BR would like to thank Hiren Patel, Pavel Fileviez Perez and Sebastian Ohmer for helpful discussions. This work has been supported in part by the Croatian Science Foundation under the project number 8799. BR acknowledges the support from the Alexander von Humboldt Foundation.

Open Access. This article is distributed under the terms of the Creative Commons Attribution License (CC-BY 4.0), which permits any use, distribution and reproduction in any medium, provided the original author(s) and source are credited.

\section{References}

[1] ATLAS collaboration, Observation of a new particle in the search for the Standard Model Higgs boson with the ATLAS detector at the LHC, Phys. Lett. B 716 (2012) 1 [arXiv: 1207.7214] [INSPIRE].

[2] CMS collaboration, Observation of a new boson at a mass of $125 \mathrm{GeV}$ with the CMS experiment at the LHC, Phys. Lett. B 716 (2012) 30 [arXiv:1207.7235] [INSPIRE].

[3] W.A. Bardeen, On naturalness in the standard model, FERMILAB-CONF-95-391 (1995).

[4] S.R. Coleman and E.J. Weinberg, Radiative Corrections as the Origin of Spontaneous Symmetry Breaking, Phys. Rev. D 7 (1973) 1888 [INSPIRE].

[5] E. Gildener and S. Weinberg, Symmetry Breaking and Scalar Bosons, Phys. Rev. D 13 (1976) 3333 [INSPIRE].

[6] K.A. Meissner and H. Nicolai, Conformal Symmetry and the Standard Model, Phys. Lett. B 648 (2007) 312 [hep-th/0612165] [INSPIRE].

[7] R. Foot, A. Kobakhidze and R.R. Volkas, Electroweak Higgs as a pseudo-Goldstone boson of broken scale invariance, Phys. Lett. B 655 (2007) 156 [arXiv:0704.1165] [INSPIRE].

[8] J.R. Espinosa and M. Quirós, Novel Effects in Electroweak Breaking from a Hidden Sector, Phys. Rev. D 76 (2007) 076004 [hep-ph/0701145] [INSPIRE].

[9] L. Alexander-Nunneley and A. Pilaftsis, The Minimal Scale Invariant Extension of the Standard Model, JHEP 09 (2010) 021 [arXiv: 1006.5916] [INSPIRE].

[10] R. Hempfling, The Next-to-minimal Coleman-Weinberg model, Phys. Lett. B 379 (1996) 153 [hep-ph/9604278] [INSPIRE].

[11] W.-F. Chang, J.N. Ng and J.M.S. Wu, Shadow Higgs from a scale-invariant hidden U(1) $(s)$ model, Phys. Rev. D 75 (2007) 115016 [hep-ph/0701254] [INSPIRE]. 
[12] S. Iso, N. Okada and Y. Orikasa, Classically conformal B - L extended Standard Model, Phys. Lett. B 676 (2009) 81 [arXiv: 0902.4050] [INSPIRE].

[13] C. Englert, J. Jaeckel, V.V. Khoze and M. Spannowsky, Emergence of the Electroweak Scale through the Higgs Portal, JHEP 04 (2013) 060 [arXiv:1301.4224] [INSPIRE].

[14] T. Hambye and A. Strumia, Dynamical generation of the weak and Dark Matter scale, Phys. Rev. D 88 (2013) 055022 [arXiv: 1306.2329] [INSPIRE].

[15] C.D. Carone and R. Ramos, Classical scale-invariance, the electroweak scale and vector dark matter, Phys. Rev. D 88 (2013) 055020 [arXiv:1307.8428] [INSPIRE].

[16] O. Antipin, M. Mojaza and F. Sannino, Conformal Extensions of the Standard Model with Veltman Conditions, Phys. Rev. D 89 (2014) 085015 [arXiv: 1310.0957] [INSPIRE].

[17] M. Hashimoto, S. Iso and Y. Orikasa, Radiative symmetry breaking at the Fermi scale and flat potential at the Planck scale, Phys. Rev. D 89 (2014) 016019 [arXiv:1310.4304] [INSPIRE].

[18] S. Abel and A. Mariotti, Novel Higgs Potentials from Gauge Mediation of Exact Scale Breaking, Phys. Rev. D 89 (2014) 125018 [arXiv:1312.5335] [INSPIRE].

[19] M. Hashimoto, S. Iso and Y. Orikasa, Radiative Symmetry Breaking from Flat Potential in various U(1)' models, Phys. Rev. D 89 (2014) 056010 [arXiv: 1401.5944] [INSPIRE].

[20] J. Kubo, K.S. Lim and M. Lindner, Electroweak Symmetry Breaking via QCD, Phys. Rev. Lett. 113 (2014) 091604 [arXiv: 1403.4262] [INSPIRE].

[21] A. Kobakhidze and K.L. McDonald, Comments on the Hierarchy Problem in Effective Theories, JHEP 07 (2014) 155 [arXiv: 1404.5823] [INSPIRE].

[22] H. Davoudiasl and I.M. Lewis, Right-Handed Neutrinos as the Origin of the Electroweak Scale, Phys. Rev. D 90 (2014) 033003 [arXiv:1404.6260] [InSPIRE].

[23] K. Kannike, A. Racioppi and M. Raidal, Embedding inflation into the Standard Model more evidence for classical scale invariance, JHEP 06 (2014) 154 [arXiv:1405.3987] [INSPIRE].

[24] M. Lindner, S. Schmidt and J. Smirnov, Neutrino Masses and Conformal Electro-Weak Symmetry Breaking, JHEP 10 (2014) 177 [arXiv:1405.6204] [INSPIRE].

[25] C.T. Hill, Is the Higgs Boson Associated with Coleman-Weinberg Dynamical Symmetry Breaking?, Phys. Rev. D 89 (2014) 073003 [arXiv:1401.4185] [INSPIRE].

[26] K. Allison, C.T. Hill and G.G. Ross, Ultra-weak sector, Higgs boson mass and the dilaton, Phys. Lett. B 738 (2014) 191 [arXiv:1404.6268] [INSPIRE].

[27] A. Farzinnia and J. Ren, Strongly First-Order Electroweak Phase Transition and Classical Scale Invariance, Phys. Rev. D 90 (2014) 075012 [arXiv:1408.3533] [INSPIRE].

[28] V.V. Khoze, C. McCabe and G. Ro, Higgs vacuum stability from the dark matter portal, JHEP 08 (2014) 026 [arXiv: 1403.4953] [InSPIRE].

[29] K. Allison, C.T. Hill and G.G. Ross, An ultra-weak sector, the strong CP problem and the pseudo-Goldstone dilaton, Phys. Lett. B (2014) [arXiv:1409.4029] [INSPIRE].

[30] T. Hambye and M.H.G. Tytgat, Electroweak symmetry breaking induced by dark matter, Phys. Lett. B 659 (2008) 651 [arXiv:0707.0633] [InSPIRE]. 
[31] R. Foot, A. Kobakhidze and R.R. Volkas, Stable mass hierarchies and dark matter from hidden sectors in the scale-invariant standard model, Phys. Rev. D 82 (2010) 035005 [arXiv: 1006.0131] [INSPIRE].

[32] K. Ishiwata, Dark Matter in Classically Scale-Invariant Two Singlets Standard Model, Phys. Lett. B 710 (2012) 134 [arXiv:1112.2696] [INSPIRE].

[33] T. Hur and P. Ko, Scale invariant extension of the standard model with strongly interacting hidden sector, Phys. Rev. Lett. 106 (2011) 141802 [arXiv:1103.2571] [INSPIRE].

[34] M. Heikinheimo, A. Racioppi, M. Raidal, C. Spethmann and K. Tuominen, Physical Naturalness and Dynamical Breaking of Classical Scale Invariance, Mod. Phys. Lett. A 29 (2014) 1450077 [arXiv: 1304.7006] [INSPIRE].

[35] M. Holthausen, J. Kubo, K.S. Lim and M. Lindner, Electroweak and Conformal Symmetry Breaking by a Strongly Coupled Hidden Sector, JHEP 12 (2013) 076 [arXiv:1310.4423] [INSPIRE].

[36] A. Farzinnia, H.-J. He and J. Ren, Natural Electroweak Symmetry Breaking from Scale Invariant Higgs Mechanism, Phys. Lett. B 727 (2013) 141 [arXiv:1308.0295] [INSPIRE].

[37] E. Gabrielli et al., Towards Completing the Standard Model: Vacuum Stability, EWSB and Dark Matter, Phys. Rev. D 89 (2014) 015017 [arXiv: 1309.6632] [INSPIRE].

[38] S. Benić and B. Radovčić, Electroweak breaking and Dark Matter from the common scale, Phys. Lett. B 732 (2014) 91 [arXiv:1401.8183] [INSPIRE].

[39] J. Guo and Z. Kang, Higgs Naturalness and Dark Matter Stability by Scale Invariance, arXiv: 1401.5609 [INSPIRE].

[40] A. Farzinnia and J. Ren, Higgs Partner Searches and Dark Matter Phenomenology in a Classically Scale Invariant Higgs Boson Sector, Phys. Rev. D 90 (2014) 015019 [arXiv: 1405.0498] [INSPIRE].

[41] J. Kubo, K.S. Lim and M. Lindner, Gamma-ray Line from Nambu-Goldstone Dark Matter in a Scale Invariant Extension of the Standard Model, JHEP 09 (2014) 016 [arXiv:1405.1052] [INSPIRE].

[42] W. Altmannshofer, W.A. Bardeen, M. Bauer, M. Carena and J.D. Lykken, Light Dark Matter, Naturalness and the Radiative Origin of the Electroweak Scale, JHEP 01 (2015) 032 [arXiv: 1408.3429] [INSPIRE].

[43] LUX collaboration, D.S. Akerib et al., First results from the LUX dark matter experiment at the Sanford Underground Research Facility, Phys. Rev. Lett. 112 (2014) 091303 [arXiv: 1310.8214] [INSPIRE].

[44] XENON1T collaboration, E. Aprile, The XENON1T Dark Matter Search Experiment, Springer Proc. Phys. C12-02-22 (2013) 93 [arXiv: 1206.6288] [INSPIRE].

[45] D.C. Malling et al., After LUX: The LZ Program, arXiv:1110.0103 [inSPIRE].

[46] B. Holdom, Two U(1)'s and Epsilon Charge Shifts, Phys. Lett. B 166 (1986) 196 [INSPIRE].

[47] C. Garcia-Cely, A. Ibarra and E. Molinaro, Dark matter production from Goldstone boson interactions and implications for direct searches and dark radiation, JCAP 11 (2013) 061 [arXiv: 1310.6256] [INSPIRE]. 
[48] J.M. Cline, G. Dupuis, Z. Liu and W. Xue, The windows for kinetically mixed $Z^{\prime}$-mediated dark matter and the galactic center gamma ray excess, JHEP 08 (2014) 131 [arXiv: 1405.7691] [INSPIRE].

[49] E.J. Chun, J.-C. Park and S. Scopel, Dark matter and a new gauge boson through kinetic mixing, JHEP 02 (2011) 100 [arXiv: 1011.3300] [INSPIRE].

[50] K. Griest, Cross-Sections, Relic Abundance and Detection Rates for Neutralino Dark Matter, Phys. Rev. D 38 (1988) 2357 [Erratum ibid. D 39 (1989) 3802] [InSPIRE].

[51] E.W. Kolb and M.S. Turner, Frontiers in Physics. Vol. 69: The Early Universe, Westview Press, Boulder U.S.A. (1990).

[52] Planck collaboration, P.A.R. Ade et al., Planck 2013 results. XVI. Cosmological parameters, Astron. Astrophys. 571 (2014) A16 [arXiv:1303.5076] [INSPIRE].

[53] D.G. Cerdeno and A.M. Green, Direct detection of WIMPs, in Particle Dark Matter, G. Bertone eds., Cambridge University Press, Cambridge U.K. (2013), pg. 347 [arXiv: 1002.1912] [INSPIRE].

[54] J.M. Cline, K. Kainulainen, P. Scott and C. Weniger, Update on scalar singlet dark matter, Phys. Rev. D 88 (2013) 055025 [arXiv: 1306.4710] [InSPIRE].

[55] M. Cirelli et al., PPPC \& DM ID: A Poor Particle Physicist Cookbook for Dark Matter Indirect Detection, JCAP 03 (2011) 051 [Erratum ibid. 1210 (2012) E01] [arXiv: 1012.4515] [INSPIRE].

[56] J. Conrad, Indirect Detection of WIMP Dark Matter: a compact review, arXiv:1411.1925 [INSPIRE]. 